\title{
Overexpression of miR-106a enhances oxaliplatin sensitivity of colorectal cancer through regulation of FOXQ1
}

\author{
ZHIHU LIU ${ }^{1}$, YAN QIN ${ }^{2}$, SHUXIAO DONG ${ }^{3}$, XIAO CHEN $^{4}$, ZHIBIN HUO $^{2}$ and ZHONGGUANG ZHEN ${ }^{1}$ \\ ${ }^{1}$ Department of Hepatobiliary Surgery; ${ }^{2}$ Surgical Department of Gastrointestinal Neoplasms, Xingtai People's Hospital; \\ ${ }^{3}$ Obstetrical Department, Xingtai Third Hospital; ${ }^{4}$ Department of Anesthesiology, \\ Xingtai People's Hospital, Xingtai, Hebei 054000, P.R. China
}

Received March 22, 2018; Accepted September 6, 2019

DOI: $10.3892 / \mathrm{ol} .2019 .11151$

\begin{abstract}
Chemotherapy resistance poses a major challenge for the clinical treatment of colorectal cancer, therefore, the aim of the present study was to examine its underlying mechanisms. Reverse transcription-quantitative polymerase chain reaction and western blot analysis were used to determine the microRNA (miRNA)/mRNA and protein expression levels, respectively. A dual luciferase assay was conducted for verification of the interaction between miR-106a and 3'untranslated region (UTR) of Forkhead box Q1 (FOXQ1). Cell viability was assessed using an MTT assay. In the present study, it was demonstrated that miR-106a is involved in regulating oxaliplatin sensitivity of colorectal cancer. Transfection of miR-106a mimics slightly inhibited colorectal cancer cell growth and sensitized colorectal cancer cells to oxaliplatin exposure. In addition, miR-106a overexpression induced a decrease of FOXQ1 at mRNA and protein levels in colorectal cancer cells. The enhanced expression of miR-106a also increased the expression of Wnt target genes, including vascular endothelial growth factor-A and matrix metallopeptidase 2, which were reported to be regulated by FOXQ1. It was predicted and validated that miR-106a could repress FOXQ1 expression via direct binding to 3'UTR. Elevation of miR-106a and a decrease of FOXQ1 expression levels were detected in tumor tissues from patients with oxaliplatin-sensitive colorectal cancer, compared with patients with oxaliplatin-resistant colorectal cancer. Furthermore, there was a significant association between miR-106a and FOXQ1 mRNA levels. In conclusion, the present study demonstrated that miR-106a increased oxaliplatin sensitivity of colorectal cancer cells through direct repression of FOXQ1 expression.
\end{abstract}

Correspondence to: Dr Zhongguang Zhen, Department of Hepatobiliary Surgery, Xingtai People's Hospital, 16 Hongxing Street, Xingtai, Hebei 054000, P.R. China

E-mail: zhenzhongguangxtph@yeah.net

Key words: microRNA-106a, oxaliplatin sensitivity, colorectal cancer, Forkhead box Q1

\section{Introduction}

Colorectal cancer is currently the third most commonly occurring cancer type in males and the second most common in females globally, with 1.4 million new cases and over 690,000 associated mortalities in 2012 (1). Oxaliplatin is one of the most frequently used drugs for the treatment of patients with colorectal cancer (2). Through the induction of intrastrand adduct formation, oxaliplatin may inhibit cell cycle progression to promote cell death in proliferative cells (3). However, de novo and acquired oxaliplatin resistance notably reduced the treatment efficacy of this drug in patients with colorectal cancer (4).

MicroRNAs (miRNAs) are small, non-coding, single-stranded RNA molecules (5). Deregulation of miRNAs has been reported to contribute to the carcinogenesis in various types of cancer, including colorectal cancer (6-8). Furthermore, a number of miRNAs, such as miR-425-5p and miR-203, have been determined to be involved in chemotherapy resistance in colorectal cancer via regulation of their target genes (9-11). Previous studies indicated that miR-106a was overexpressed in tumor tissues, fecal samples and plasma of patients with colorectal cancer (12-14). A decrease in miR-106a levels has been previously reported to predict a reduced disease-free survival (DFS) and overall survival (OS) times in patients with colorectal cancer (15), suggesting a dual role of miR-106a in colorectal cancer.

Forkhead box Q1 (FOXQ1) is a member of the FOX gene family and functions as a transcription factor (16). Accumulating evidence has indicated that FOX proteins serve as terminal effectors for numerous signaling pathways, including the transforming growth factor- $\beta$ signaling, Wnt, Hedgehog and mitogen-activated protein kinase pathways (17). Previously, FOXQ1 was determined to be overexpressed in colorectal cancer and promoted colorectal cancer progression $(18,19)$. A limited number of studies have indicated that FOXQ1 may be regulated by transcriptional activation, miRNA binding and post-translational modification under different conditions (20-22). For example, in colorectal cancer cells, FOXQ1 was identified as a target gene of Wnt signaling pathway (20). However, the specific underlying mechanism of the deregulation of FOXQ1 by miRNA in colorectal cancer remains unknown. 
In the present study, the function of miR-106a in mediating oxaliplatin sensitivity in colorectal cancer was investigated. Overexpression of miR-106a slightly decreased cell growth ability and sensitized colorectal cancer cells to oxaliplatin treatment. Reverse transcription-quantitative polymerase chain reaction (RT-qPCR) and western blot analysis demonstrated that miR-106a could downregulate FOXQ1 at mRNA and protein levels in colorectal cancer cells. The dual luciferase reporter assay confirmed that FOXQ1 was a direct target of miR-106a. Furthermore, it was observed that there was a decrease in miR-106a expression levels and an increase in FOXQ1 mRNA expression levels in tumor tissues from patients with oxaliplatin-resistant colorectal cancer, compared with patients with oxaliplatin-sensitive colorectal cancer. In addition, a significant association between miR-106a and FOXQ1 expression in tumor tissues was observed. The present study identified miR-106a as a promising target for oxaliplatin resistance in colorectal cancer.

\section{Materials and methods}

Patients. In the present study, the gene expression of 30 patients with colorectal cancer, including 17 patients (11 men and

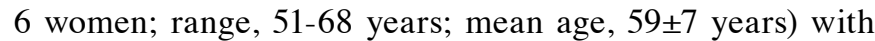
oxaliplatin-sensitive colorectal cancer and 13 patients (8 males and 5 females; range 56-69 years) with oxaliplatin-resistant colorectal cancer were examined. All the participants received oxaliplatin-based chemotherapy and were enrolled in Xingtai People's Hospital (Xingtai, China) between February 2013 and March 2015. Tumor response and progression were assessed according to the Response Evaluation Criteria in Solid Tumors version 1.1 (23). According to the outcome of therapy, 17 patients were classified as responders (complete or partial response) and 13 patients were classified as non-responders (no change and progressive disease). Written consents regarding participation in the present study and use of their tissues were obtained from all patients and the experiments were conducted with the approval of and under the supervision of the Ethics Committee of Xingtai People's Hospital.

Cell culture and oxaliplatin treatment. The kidney derived 293T cell line and human colorectal cancer cell lines, HCT116 and HT-29, which were commonly used to study oxaliplatin sensitivity of colorectal cancer (24-25), were obtained from the American Type Culture Collection and used within 6 months. HT-29 cell line was authenticated by STR profiling. The HCT116 and HT-29 cell lines were maintained in Dulbecco's modified Eagle's medium (Gibco; Thermo Fisher Scientific, Inc.) containing $10 \%$ fetal bovine serum (Gibco; Thermo Fisher Scientific, Inc.) in an incubator at $37^{\circ} \mathrm{C}$ with $5 \% \mathrm{CO}_{2}$.

For oxaliplatin treatment, indicated concentrations (2, 4,6 and $8 \mu \mathrm{mol} / \mathrm{l}$ ) of oxaliplatin (Selleck Chemicals) were added into the culture medium of HCT116 and HT-29 cells and sustained for $48 \mathrm{~h}$ at $37^{\circ} \mathrm{C}$ then subjected to the following procedures.

MTT assay. Cell viability was assessed with an MTT assay. Briefly, HCT116 and HT-29 cells were cultured at $37^{\circ} \mathrm{C}$ in 96-well plates $\left(1 \times 10^{3}\right.$ cells/well) and treated with oxaliplatin for the indicated lengths of time $(0,25,50,75$ and $100 \mathrm{~h})$.
Subsequently, HCT116 and HT-29 cells were stained with thiazolyl blue tetrazolium bromide (MTT; Sigma-Aldrich; Merck $\mathrm{KGaA}$ ) for $2 \mathrm{~h}$ at $37^{\circ} \mathrm{C}$ and absorbance at $570 \mathrm{~nm}$ was detected following purple precipitates being dissolved with MTT detergent reagent on a microplate reader (BioTek Instruments, Inc.). Cell viability was calculated as the ratio of the absorbance values of treated samples to those of controls.

RNA extraction and $R T-q P C R$. RNA was extracted from HCT116 and HT-29 cells and tissues from patients using TRIzol $^{\circledR}$ (Invitrogen; Thermo Fisher Scientific, Inc.), according to the manufacturer's protocol. Total RNA was reverse transcribed into cDNA with a PrimeScript ${ }^{\mathrm{TM}}$ RT reagent kit (Takara Bio, Inc.), according to manufacturer's protocol. For mRNA expression analysis of FOXQ1, the RT-qPCR was performed on a CFX96 Real-Time PCR Detection system (Bio-Rad Laboratories, Inc.) with SYBR ${ }^{\circledR}$ Premix Ex Taq ${ }^{\mathrm{TM}}$ II (Takara Bio, Inc.). GAPDH was used as an endogenous reference gene.

For miRNA expression analysis, RT was performed with a miScript II RT kit (Qiagen $\mathrm{GmbH}$ ). Expression levels of miR-106a were detected using miScript primer assays with a miScript SYBR Green PCR kit (Qiagen GmbH), according to the manufacturer's protocol. U6 was used as an endogenous reference gene. The thermocycling conditions were as follows: $95^{\circ} \mathrm{C}$ for $15 \mathrm{~min}$, followed by denaturation: $95^{\circ} \mathrm{C}$ for $10 \mathrm{sec}$, annealing for $30 \mathrm{sec}$ and extension for $30 \mathrm{sec}$ ( 40 cycles). The relative expression of miRNA and mRNA were calculated using $2^{-\Delta \Delta \mathrm{Cq}}$ method (26). The primer sequences were listed as follows: FOXQ1-Forward: 5'-CACGCAGCAAGCCAT ATACG-3'; FOXQ1-Reverse: 5'-CGTTGAGCGAAAGGT TGTGG-3'; MMP-2-Forward: 5'-GGCCCTGTCACTCCT GAGAT-3'; MMP-2-Reverse: 5'-GGCATCCAGGTTATC GGGGA-3'; VEGF-A-Forward: 5'-AGGGCAGAATCATCA CGAAGT-3'; VEGF-A-Reverse: 5'-AGGGTCTCGATTGGA TGGCA-3'; GAPDH-Forward: 5'-CTCTGATTTGGTCGT ATTGGG-3'; GAPDH-Reverse: 5'-TGGAAGATGGTGATG GGATT-3'; miR-106a-RT: 5'-GTCGTATCCAGTGCGTGT CGTGGAGTCGGCAATTGCACTGGATACGACCTACCT G-3'; miR-106a-Forward: 5'-ATCCAGTGCGTGTCGTG-3'; miR-106a-Reverse: 5'-TGCTAAAAGTGCTTACAGTG-3'; U6-Forward: 5'-CTCGCTTCGGCAGCACA-3'; U6-Reverse: 5'-AACGCTTCACGAATTTGCGT-3'.

Western blot analysis. The FOXQ1 antibody (cat. no. SC-166265; dilution, 1:2,000) and vascular endothelial growth factor-A (VEGF-A; cat. no. SC-365578; dilution, 1:2,000) were purchased from Santa Cruz Biotechnology, Inc. Antibodies against matrix metallopeptidase-2 (MMP-2; cat. no. 40994; dilution, 1:1,000) was purchased from Cell Signaling Technology, Inc. The GAPDH antibody was obtained from Sigma-Aldrich (Merck KGaA). The secondary antibodies against mouse and rabbit (HRP-conjugate; dilution, 1:10,000) were purchased from ProteinTech Group, Inc. Proteins were extracted from HCT116 and HT-29 cells using radioimmunoprecipitation assay lysis buffer (Sigma-Aldrich; Merck KGaA). The concentration of protein lysates was determined by bicinchoninic acid assay (BCA assay) using a BCA protein assay kit (Thermo Fisher Scientific, Inc.), according to the manufacturer's protocol. Western blot analysis was 
A

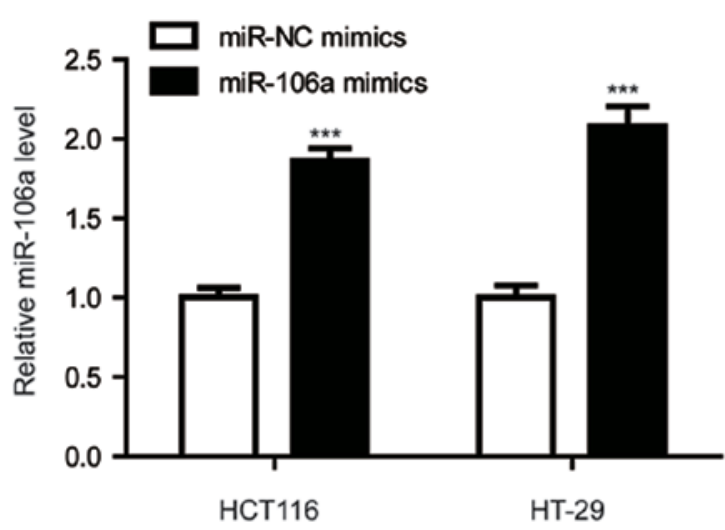

C

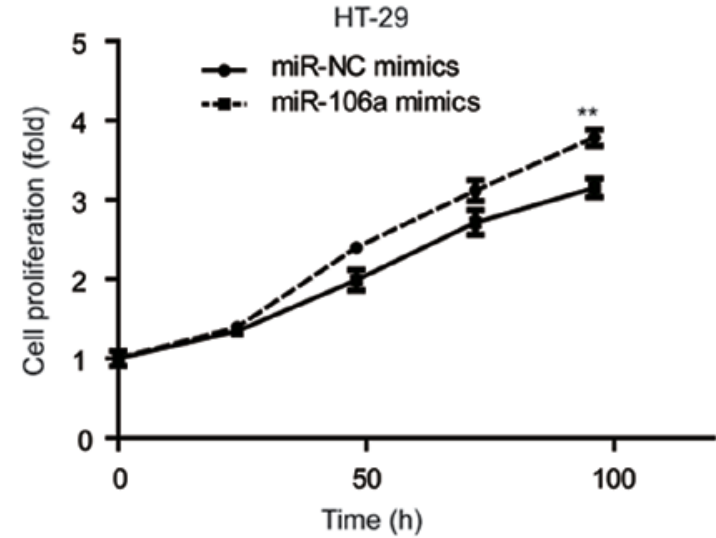

B

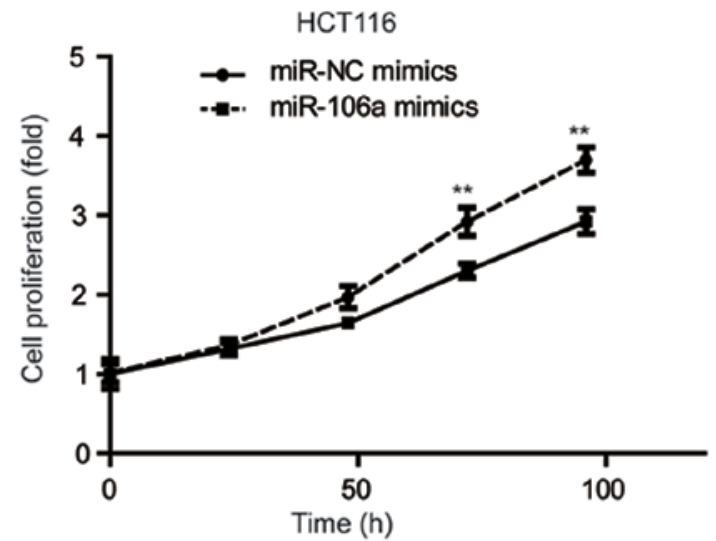

HCT116

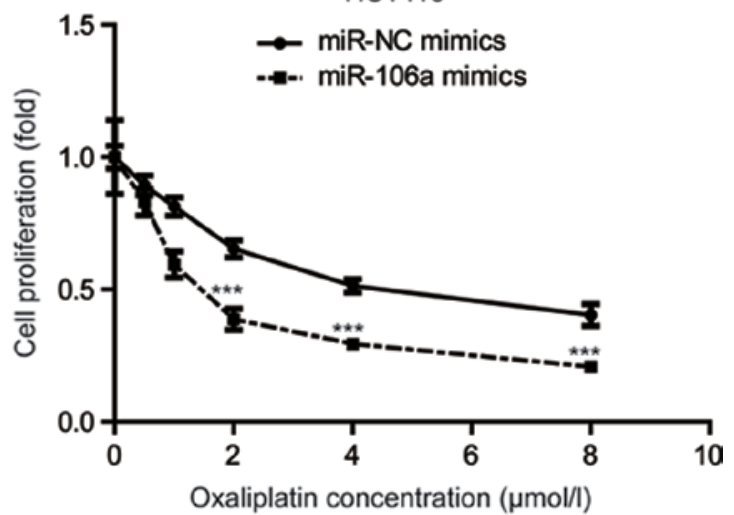

$E$

HT-29

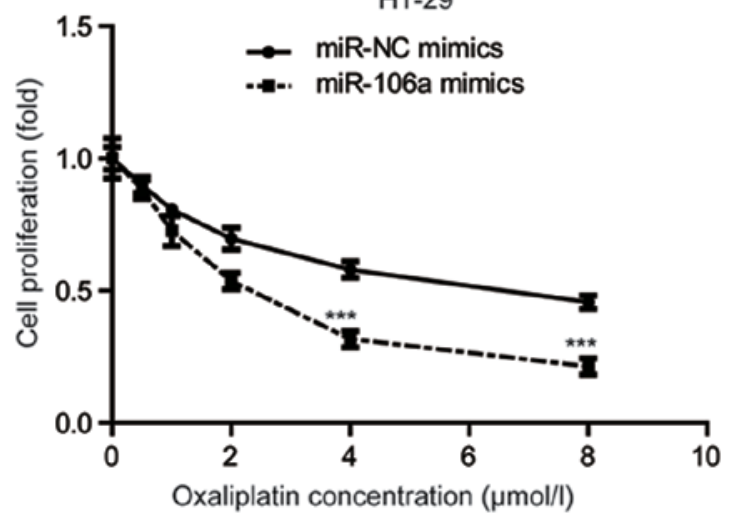

Figure 1. Enhanced expression of miR-106a sensitizes colorectal cancer cells to oxaliplatin treatment. (A) Transfection of miR-106a mimics increased miR-106a expression levels in HCT116 and HT-29 cells. (B) MTT assay demonstrated that overexpression of miR-106a enhanced cell proliferation ability of HCT-116 cells. (C) miR-106a mimics increased cell proliferation ability of HT-29 cells. (D) Results from cell proliferation assay demonstrated that miR-106a-overexpressing colorectal cancer cells had increased sensitivity to increasing treatment of oxaliplatin in the HCT-116 cell line. (E) Results from cell proliferation assay demonstrated that miR-106a-overexpressing colorectal cancer cells had increased sensitivity to treatment of increasing concentrations of oxaliplatin in the HT-29 cell line. ${ }^{* *} \mathrm{P}<0.01$ and ${ }^{* * *} \mathrm{P}<0.001$, miR-106a mimics vs. miR-NC mimics. miR, microRNA; NC, negative control.

performed as follows: Equal amounts $(20 \mu \mathrm{g})$ of samples were loaded into each lane on an $8 \%$ SDS-PAGE gel. The proteins were separated and transferred on a polyvinylidene fluoride membrane. Following blocking in 5\% non-fat milk for $1 \mathrm{~h}$ at room temperature, the membranes were incubated with primary antibodies overnight at $4^{\circ} \mathrm{C}$. Subsequently, the membranes were incubated with secondary antibodies for $1 \mathrm{~h}$ at room temperature and then developed with Enhanced Chemiluminescent Western Blotting Substrate (Pierce; Thermo Fisher Scientific, Inc.). Images were captured with ImageQuant LAS 4000 (GE Healthcare Life Sciences). GAPDH was used as endogenous control.
Transfection of miR-Negative Control (miR-NC) mimics and miR-106a mimics. miR-NC mimics and miR-106a mimics were purchased from Shanghai GenePharma Co., Ltd. The sequences were: miR-NC mimics, 5'-UUCUCCGAACGU GUCACGUTT-3'; miR-106a mimics, 5'-AAAAGUGCUUAC AGUGCAGGUAG-3'. miR-NC mimics or miR-106a mimics were transfected at a final concentration of $40 \mathrm{nM}$ with Lipofectamine ${ }^{\circledR} 2000$ (Invitrogen; Thermo Fisher Scientific, Inc.), according to the manufacturer's protocols. In brief, miR-NC mimics or miR-106a mimics and Lipofectamine ${ }^{\circledR}$ 2000 were mixed (to a concentration of $200 \mathrm{nM}$ ) in Opti-MEM medium (Thermo Fisher Scientific, Inc.) and incubated 


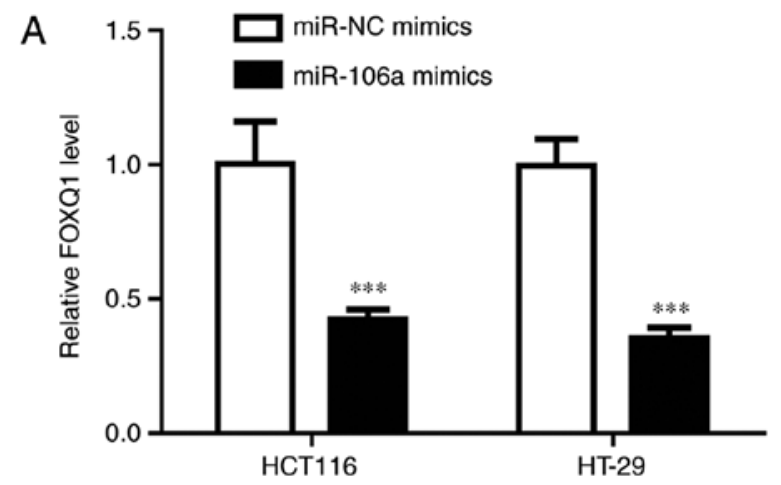

B

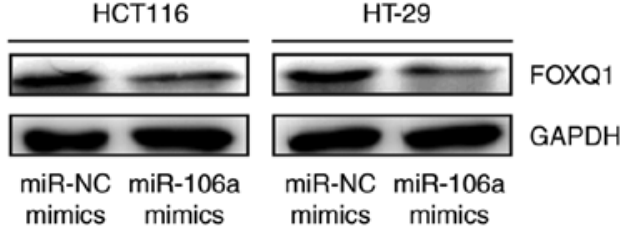

Figure 2. miR-106a represses FOXQ1 expression in colorectal cancer cells. (A) Transfection of miR-106a mimics reduced FOXQ1 mRNA levels in colorecta cancer cells. (B) Transfection of miR-106a mimics reduced FOXQ1 protein levels in colorectal cancer cells. ${ }^{* * *} \mathrm{P}<0.001$, miR-106a mimics vs. miR-NC mimics. miR, microRNA; FOXQ1, Forkhead box Q1.

A

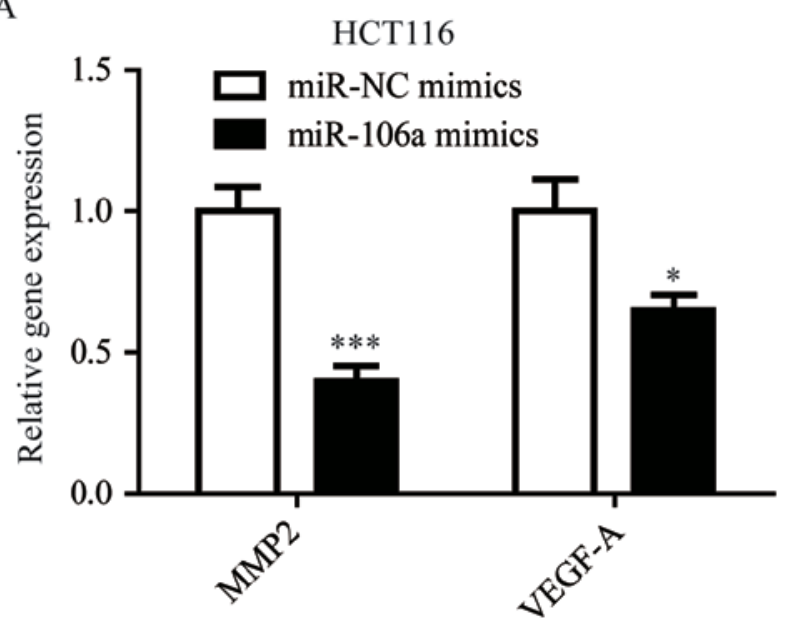

C

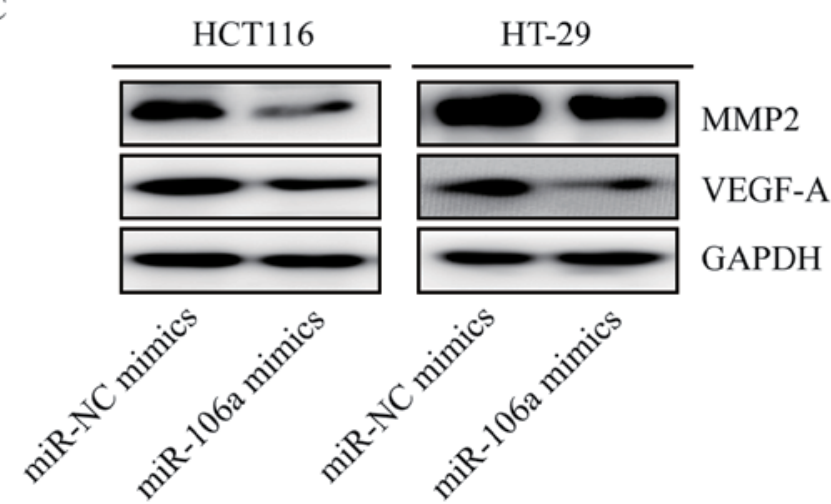

B

HT-29

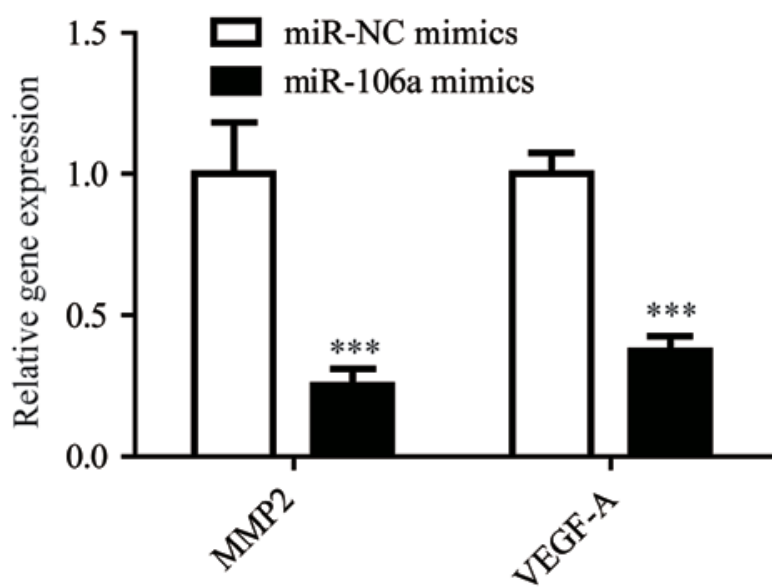

Figure 3. Overexpression of miR-106a decreases FOXQ1 target gene expression. (A) Transfection of miR-106a mimics reduced VEGF-A and MMP-2 mRNA levels in colorectal cancer cell lines HCT116. (B) Transfection of miR-106a mimics reduced VEGF-A and MMP-2 protein levels in colorectal cancer HT-29 cell line. (C) Protein expression levels of VEGF-A and MMP-2 were reduced in response to miR-106a overexpression in colorectal cancer cell lines HCT116 and HT-29. ${ }^{*} \mathrm{P}<0.05$ and ${ }^{* * *} \mathrm{P}<0.001$, miR-106a mimics vs. miR-NC mimics. miR, microRNA; FOXQ1, Forkhead box Q1; VEGF-A, vascular endothelial growth factor-A; MMP-2, matrix metallopeptidase-2.

for $20 \mathrm{~min}$ at $37^{\circ} \mathrm{C}$. Subsequently, the mixture was added to HCT116 and HT-29 cells in DMEM. The medium was replaced with fresh DMEM after $6 \mathrm{~h}$ and sustained for $18 \mathrm{~h}$ before subjected to following experiments.

Dual luciferase reporter assay. Sequencing alignment was performed using miRDB database (www.mirdb.org). FOXQ1
3'UTR was amplified from cDNA of 293T cells and ligated into a pGL3 plasmid (Promega Corporation). A total of two-point mutations were introduced into FOXQ1 3'UTR with a Site-directed mutagenesis kit (Agilent Technologies, Inc.), in order to construct pGL3-FOXQ1-3'UTR-Mut.

For the dual luciferase reporter assay, 293T and HCT116 cells were plated on 24-well plates. On the following 
day, cells were co-transfected with pGL3-FOXQ1-WT or pGL3-FOXQ1-3'UTR-Mut, miR-NC mimics or miR-106a mimics and pRL-TK plasmid (Invitrogen; Thermo Fisher Scientific, Inc.) using Lipofectamine ${ }^{\circledR} 2000$. The assay was performed $48 \mathrm{~h}$ after with a Dual-luciferase Assay system (Promega Corporation). The firefly luciferase activity was normalized to the Renilla luciferase activity value.

Statistical analysis. The data were calculated with GraphPad Prism v6 (GraphPad Software, Inc.) and presented as the mean \pm standard deviation. Comparisons between two different groups were determined with unpaired Student's t-test. $\mathrm{P}<0.05$ was considered to indicate a statistically significant difference.

\section{Results}

Overexpression of miR-106a-transfected oxaliplatin-sensitized colorectal cancer cells. In order to evaluate the role of miR-106a in colorectal cancer cells, the HCT116 and HT-29 cell lines were selected. miR-106a was significantly overexpressed in these cell lines via transfection of miR-106a mimics $(\mathrm{P}<0.001$; Fig. 1A). A cell viability assay was conducted to detect cell proliferation ability upon miR-106a overexpression. As depicted in Fig. 1B and C, miR-106a mimics significantly increased MTT absorbance compared with cells transfected with miR-NC mimics $(\mathrm{P}<0.01)$, indicating miR-106a overexpression promoted cell proliferation of HCT116 and HT-29 cells. However, in cells treated with increasing concentrations of oxaliplatin, overexpression of miR-106a significantly reduced cell viability, compared with control groups, in the cell lines tested $(\mathrm{P}<0.001$; Fig. 1D and $\mathrm{E})$. These results indicated that miR-106a overexpression could enhance oxaliplatin sensitivity in colorectal cancer cells.

miR-106a represses FOXQ1 expression in colorectal cancer cells. FOXQ1 is frequently overexpressed in colorectal cancer and its expression is associated with oxaliplatin resistance (27). Transfection of miR-106a mimics significantly decreased FOXQ1 mRNA level in HCT116 and HT-29 cells $(\mathrm{P}<0.001$; Fig. 2A). Western blot analysis demonstrated that the protein expression level of FOXQ1 was also reduced following miR-106 overexpression (Fig. 2B). These results indicated that miR-106a may regulate oxaliplatin sensitivity via repression of FOXQ1 level.

miR-106a regulates FOXQ1 target genes. FOXQ1 is a transcriptional factor and can activate VEGF-A and MMP-2 transcription via regulation of the Wnt signaling pathway in colorectal cancer (27). In addition, overexpression of miR-106a significantly reduced VEGF-A $(\mathrm{P}<0.05)$ and MMP-2 $(\mathrm{P}<0.001)$ mRNA in HCT116 and HT-29 cells (Fig. 3A and B), and the protein levels of VEGF-A and MMP-2 were also reduced in the two cell lines (Fig. 3C). These results indicated that miR-106a could inhibit FOXQ1, and repress transcription of its target genes.

miR-106a directly inhibits FOXQ1 expression. The aim of the present study was to investigate whether miR-106a directly regulates FOXQ1 expression. Sequence alignment demonstrated that there were binding sites for miR-106a in the 3'UTR
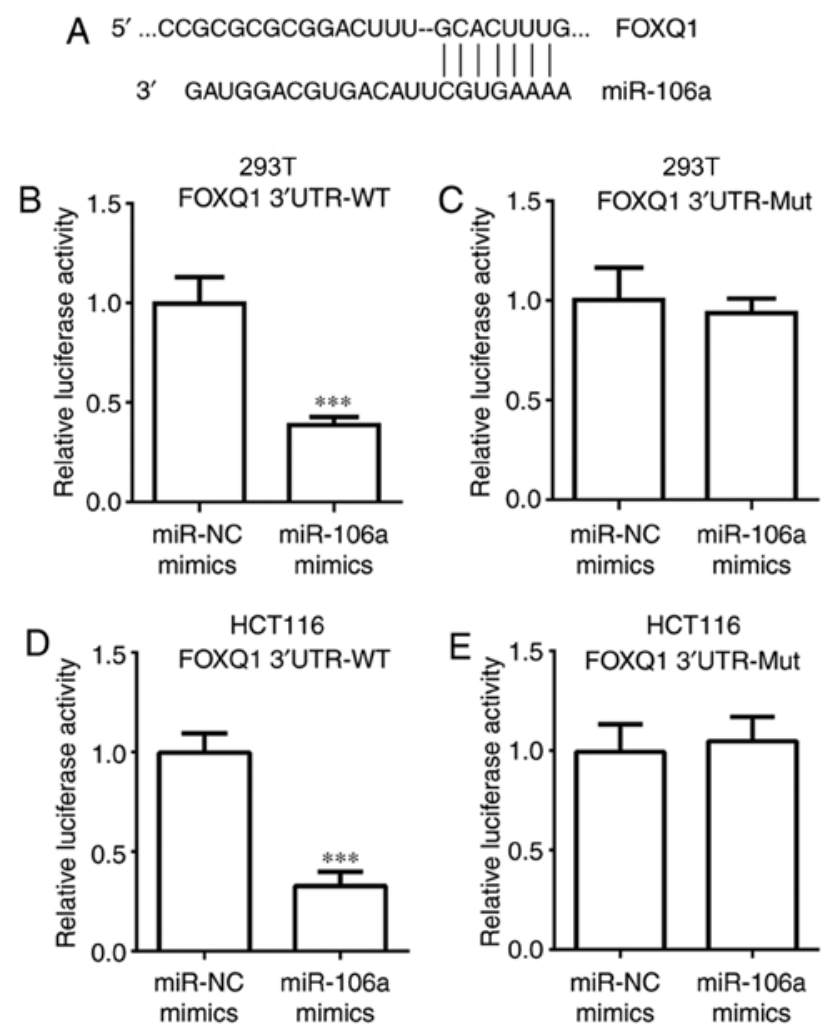

Figure 4. FOXQ1 is a direct target of miR-106a. (A) Sequence alignment of miR-106a with 3'UTR of FOXQ1 mRNA was exhibited. (B) Transfection of miR-106a mimics reduced luciferase activity of $293 \mathrm{~T}$ cells transfected with FOXQ1 3'UTR-WT. (C) Transfection of miR-106a mimics did not alter luciferase activity of 293T cells transfected with pGL3-FOXQ1-3'UTR-Mut (D) Transfection of miR-106a mimics reduced luciferase activity of HCT116 cells transfected with FOXQ1 3'UTR-WT. (E) Transfection of miR-106a mimics did not alter luciferase activity of HCT-116 cells transfected with pGL3-FOXQ1-3'UTR-Mut. ${ }^{* * *} \mathrm{P}<0.001$, miR-106a mimics vs. miR-NC mimics. miR, microRNA; FOXQ1, Forkhead box Q1; UTR, untranslated region.

of FOXQ1 mRNA (Fig. 4A). In the dual luciferase assay, it was determined that transfection of miR-106a mimics significantly decreased relative luciferase activity of $293 \mathrm{~T}$ cells transfected with FOXQ1 3'UTR-WT, but not FOXQ1 3'UTR-Mut ( $\mathrm{P}<0.001$; Fig. 4B and C). Similar results were observed in HCT116 cells $(\mathrm{P}<0.001$; Fig. 4D and E). These data confirmed that miR-106a could bind to FOXQ1 mRNA and directly repress its expression.

Expression of miR-106a and FOXQ1 in tumor tissues from patients with colorectal cancer. To further evaluate the role of miR-106a and FOXQ1 in oxaliplatin sensitivity, the expression of miR-106a and FOXQ1 in tumor tissues from patients with colorectal cancer receiving oxaliplatin-based chemotherapy was determined. RT-qPCR demonstrated that miR-106a expression levels $(\mathrm{P}<0.05)$ were significantly decreased in tumor tissues from oxaliplatin non-responders, while FOXQ1 $(\mathrm{P}<0.001)$ mRNA levels were significantly elevated (Fig. 5A and B). Consistent with the present in vitro data, statistical analysis indicated that the expression of miR-106a was associated with FOXQ1 expression ( $\mathrm{r}=-0.5535 ; \mathrm{P}=0.0015$; Fig. 5C). These results demonstrated that miR-106a regulates the expression of FOXQ1 in tumor tissues of patients with colorectal cancer. 
A

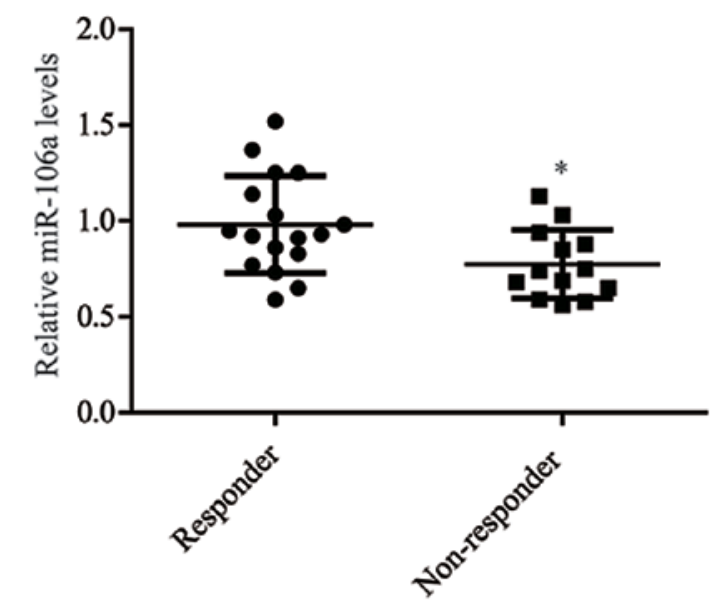

$\mathrm{C}$

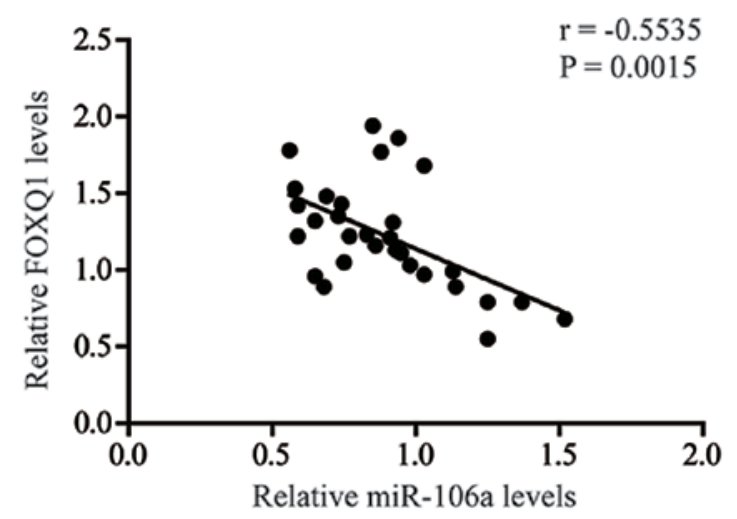

B

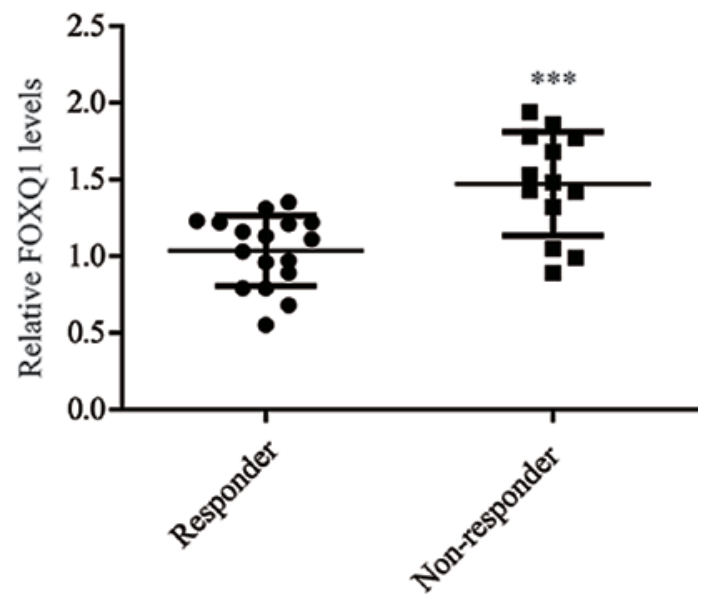

Figure 5. Expression of FOXQ1 and miR-106a in tumor tissues from patients with colorectal cancer are presented. (A) Compared with colorectal tumor tissues from responders, miR-106a expression was decreased in tumor tissues from non-responders. (B) Compared with colorectal tumor tissues from responders, FOXQ1 expression was elevated in tumor tissues from non-responders. (C) There was a significant negative association between miR-106a and FOXQ1 expression levels observed in tumor tissues from patients with colorectal cancer. ${ }^{*} \mathrm{P}<0.05$ and ${ }^{* * *} \mathrm{P}<0.001$, non-responder vs. responder. miR, microRNA; FOXQ1, Forkhead box Q1.

\section{Discussion}

Oxaliplatin is one of the commonly used chemotherapy agents for patients with cancer (28). Resistance to oxaliplatin frequently occurs in the clinical management of colorectal cancer (29). The underlying mechanism of oxaliplatin resistance is complicated and includes dysregulation of numerous miRNAs and proteins, including upregulation of miR-203 (10,30,31). In the present study, miR-106a was identified as an oxaliplatin sensitizer in colorectal cancer cells via regulation of FOXQ1 expression.

Deregulation of miRNAs was a signature and early event in the initiation and development of colorectal cancer (32). Previous findings demonstrated that miR-106a was overexpressed in colorectal cancer and predicted poor prognosis $(15,33,34)$. miR-106a could regulate cell proliferation, apoptosis, migration and invasion in colon cancer cells via repression of its target genes $(12,35)$. In a recent meta-analysis, a high expression of miR-106a was found to be associated with poor overall survival of colorectal cancer patients (36). In the present study, it was validated that miR-106a overexpression promoted colorectal cancer cell growth. Notably, overexpression of miR-106a sensitized colorectal cancer cells to oxaliplatin treatment. Additionally, the expression of miR-106a was reduced in tumor tissues from non-responder patients with colorectal cancer, compared with responders. These data indicated a role of miR-106a in regulating oxaliplatin sensitivity in colorectal cancer cells.

FOXQ1 is overexpressed in colorectal cancer types (37). In colorectal cancer cells, it was determined that miR-106a overexpression resulted in decreased FOXQ1 level. A dual luciferase assay was conducted to further confirm FOXQ1 as a direct target of miR-106a. As a transcription factor, FOXQ1 could activate target gene expression in order to regulate cell growth, migration, epithelial-mesenchymal transition and chemoresistance in colorectal cancer cells $(18,38)$. Western blot analysis and RT-qPCR demonstrated that the expression of FOXQ1 target genes were also repressed in colorectal cancer cells with overexpressed miR-106a. Due to miR-106a repressing FOXQ1 expression, it was concluded that miR-106a may control oxaliplatin sensitivity via the regulation of FOXQ1 in colorectal cancer. Furthermore, FOXQ1 expression was significantly increased in colorectal tumor tissues from non-responders, compared with responders, and an association was indicated between FOXQ1 and miR-106a expression levels in colorectal tumor tissues. Collectively, these results demonstrated that miR-106a could sensitize colorectal cancer cells to oxaliplatin. 
The present results indicated that miR-106a serves an important role in determining the sensitivity of colorectal cancer cells to oxaliplatin treatment. The present study improved the understanding of the role of miR-106a in colorectal cancer and may allow for the use of miR-106a as a biomarker and target for patients receiving chemotherapy, facilitating the development of novel chemotherapy strategies.

The present study focused on the expression of miR-106a in tumor tissues from oxaliplatin responder and oxaliplatin non-responder. miR-106a expression in colorectal normal tissues and tumor tissues and its role in regulating colorectal cancer cell proliferation will be further evaluated in the future.

\section{Acknowledgements}

Not applicable.

\section{Funding}

No funding was received.

\section{Availability of data and materials}

The datasets used and/or analyzed during the present study are available from the corresponding author on reasonable request.

\section{Authors' contributions}

ZL, YQ and SD performed the experiments, analyzed the data and wrote the manuscript. YQ and SD participated in the collection of patient samples. $\mathrm{XC}$ and $\mathrm{ZH}$ assisted with the western blot analysis and data collection. ZZ conceived the idea, designed the study and helped to draft the manuscript. All authors read and approved the final manuscript.

\section{Ethics approval and consent to participate}

The present study was approved by the Ethic Committee of Xingtai People's Hospital. All subjects provided written informed consent prior to enrollment.

\section{Patient consent for publication}

Not applicable.

\section{Competing interests}

The authors declare that they have no competing interests.

\section{References}

1. Torre LA, Bray F, Siegel RL, Ferlay J, Lortet-Tieulent J and Jemal A: Global cancer statistics, 2012. CA Cancer J Clin 65: 87-108, 2015.

2. André T, Boni C, Mounedji-Boudiaf L, Navarro M, Tabernero J, Hickish T, Topham C, Zaninelli M, Clingan P, Bridgewater J, et al: Oxaliplatin, fluorouracil, and leucovorin as adjuvant treatment for colon cancer. N Engl J Med 350: 2343-2351, 2004.

3. Raymond E, Faivre S, Chaney S, Woynarowski J and Cvitkovic E: Cellular and molecular pharmacology of oxaliplatin. Mol Cancer Ther 1: 227-235, 2002.

4. Rabik CA and Dolan ME: Molecular mechanisms of resistance and toxicity associated with platinating agents. Cancer Treat Rev 33: 9-23, 2007.
5. Ambros V: microRNAs: Tiny regulators with great potential. Cell 107: 823-826, 2001.

6. Ardekani AM and Naeini MM: The role of microRNAs in human diseases. Avicenna J Med Biotechnol 2: 161-179, 2010.

7. Du B, Wu D, Yang X, Wang T, Shi X, Lv Y, Zhou Z, Liu Q and Zhang W: The expression and significance of microRNA in different stages of colorectal cancer. Medicine (Baltimore) 97: e9635, 2018.

8. Emami SS, Akbari A, Zare AA, Agah S, Masoodi M, Talebi A, Minaeian S, Fattahi A and Moghadamnia F: MicroRNA expression levels and histopathological features of colorectal cancer. J Gastrointest Cancer 50: 276-284, 2019.

9. Zhang Y, Hu X, Miao X, Zhu K, Cui S, Meng Q, Sun J and Wang T: MicroRNA-425-5p regulates chemoresistance in colorectal cancer cells via regulation of programmed cell death 10. J Cell Mol Med 20: 360-369, 2016.

10. Zhou Y, Wan G, Spizzo R, Ivan C, Mathur R, Hu X, Ye X, Lu J, Fan F, Xia L, et al: miR-203 induces oxaliplatin resistance in colorectal cancer cells by negatively regulating ATM kinase. Mol Oncol 8: 83-92, 2014.

11. Ayers D and Vandesompele J: Influence of microRNAs and long non-coding RNAs in cancer chemoresistance. Genes (Basel) 8: pii: E95, 2017.

12. Feng B, Dong TT, Wang LL, Zhou HM, Zhao HC, Dong F and Zheng MH: Colorectal cancer migration and invasion initiated by microRNA-106a. PLoS One 7: e43452, 2012.

13. Koga Y, Yamazaki N, Yamamoto Y, Yamamoto S, Saito N, Kakugawa Y, Otake Y, Matsumoto M and Matsumura Y: Fecal miR-106a is a useful marker for colorectal cancer patients with false-negative results in immunochemical fecal occult blood test. Cancer Epidemiol Biomarkers Prev 22: 1844-1852, 2013.

14. Liu GH, Zhou ZG, Chen R, Wang MJ, Zhou B, Li Y and Sun XF: Serum miR-21 and miR-92a as biomarkers in the diagnosis and prognosis of colorectal cancer. Tumour Biol 34: 2175-2181, 2013

15. Diaz R, Silva J, Garcia JM, Lorenzo Y, García V, Peña C, Rodríguez R, Muñoz C, García F, Bonilla F and Domínguez G: Deregulated expression of miR-106a predicts survival in human colon cancer patients. Genes Chromosomes Cance 47: 794-802, 2008.

16. Overdier DG, Porcella A and Costa RH: The DNA-binding specificity of the hepatocyte nuclear factor 3/forkhead domain is influenced by amino-acid residues adjacent to the recognition helix. Mol Cell Biol 14: 2755-2766, 1994.

17. Zhu H: Targeting forkhead box transcription factors FOXM1 and FOXO in leukemia (Review). Oncol Rep 32: 1327-1334, 2014

18. Kaneda H, Arao T, Tanaka K, Tamura D, Aomatsu K, Kudo K, Sakai K, De Velasco MA, Matsumoto K, Fujita Y, et al: FOXQ1 is overexpressed in colorectal cancer and enhances tumorigenicity and tumor growth. Cancer Res 70: 2053-2063, 2010.

19. Weng W, Okugawa Y, Toden S, Toiyama Y, Kusunoki M and Goel A: FOXM1 and FOXQ1 are promising prognostic biomarkers and novel targets of tumor-suppressive miR-342 in human colorectal cancer. Clin Cancer Res 22: 4947-4957, 2016.

20. Christensen J, Bentz S, Sengstag T, Shastri VP and Anderle P: FOXQ1, a novel target of the Wnt pathway and a new marker for activation of Wnt signaling in solid tumors. PLoS One 8: e60051, 2013.

21. Vishnubalaji R, Hamam R, Yue S, Al-Obeed O, Kassem M, Liu FF, Aldahmash A and Alajez NM: MicroRNA-320 suppresses colorectal cancer by targeting SOX4, FOXM1, and FOXQ1. Oncotarget 7: 35789-35802, 2016.

22. Calnan DR and Brunet A: The FoxO code. Oncogene 27: 2276-2288, 2008.

23. Eisenhauer EA, Therasse P, Bogaerts J, Schwartz LH, Sargent D, Ford R, Dancey J, Arbuck S, Gwyther S, Mooney M, et al: New response evaluation criteria in solid tumours: Revised RECIST guideline (version 1.1). Eur J Cancer 45: 228-247, 2009.

24. Codony-Servat J, Cuatrecasas M, Asensio E, Montironi C, Martínez-Cardús A, Marín-Aguilera M, Horndler C, Martínez-Balibrea E, Rubini M, Jares P, et al: Nuclear IGF-1R predicts chemotherapy and targeted therapy resistance in metastatic colorectal cancer. Br J Cancer 117: 1777-1786, 2017.

25. Ju HQ, Lu YX, Wu QN, Liu J, Zeng ZL, Mo HY, Chen Y, Tian T, Wang Y, Kang TB, et al: Disrupting G6PD-mediated Redox homeostasis enhances chemosensitivity in colorectal cancer. Oncogene 36: 6282-6292, 2017.

26. Livak KJ and Schmittgen TD: Analysis of relative gene expression data using real-time quantitative PCR and the 2(-Delta Delta C(T)) method. Methods 25: 402-408, 2001. 
27. Peng X, Luo Z, Kang Q, Deng D, Wang Q, Peng H, Wang S and Wei Z: FOXQ1 mediates the crosstalk between TGF- $\beta$ and Wnt signaling pathways in the progression of colorectal cancer. Cancer Biol Ther 16: 1099-1109, 2015.

28. Davies JM and Goldberg RM: First-line therapeutic strategies in metastatic colorectal cancer. Oncology (Williston Park) 22: 1470-1479, 2008.

29. Hector S, Bolanowska-Higdon W, Zdanowicz J, Hitt S and Pendyala L: In vitro studies on the mechanisms of oxaliplatin resistance. Cancer Chemother Pharmacol 48: 398-406, 2001.

30. Li J, Chen Y, Zhao J, Kong F and Zhang Y: miR-203 reverses chemoresistance in p53-mutated colon cancer cells through downregulation of Akt2 expression. Cancer Lett 304: 52-59, 2011.

31. Sau A, Pellizzari Tregno F, Valentino F, Federici G and Caccuri AM: Glutathione transferases and development of new principles to overcome drug resistance. Arch Biochem Biophys 500: 116-122, 2010.

32. Thomas J, Ohtsuka M, Pichler M and Ling H: MicroRNAs: Clinical relevance in colorectal cancer. Int J Mol Sci 16: 28063-28076, 2015.

33. Bovell LC, Shanmugam C, Putcha BD, Katkoori VR, Zhang B, Bae S, Singh KP, Grizzle WE and Manne U: The prognostic value of microRNAs varies with patient race/ethnicity and stage of colorectal cancer. Clin Cancer Res 19: 3955-3965, 2013.
34. Li J, Liu Y, Wang C, Deng T, Liang H, Wang Y, Huang D, Fan Q, Wang $\mathrm{X}$, Ning T, et al: Serum miRNA expression profile as a prognostic biomarker of stage II/III colorectal adenocarcinoma. Sci Rep 5: 12921, 2015.

35. Qin Y, Huo Z, Song X, Chen X, Tian X and Wang X: mir-106a regulates cell proliferation and apoptosis of colon cancer cells through targeting the PTEN/PI3K/AKT signaling pathway. Oncol Lett 15: 3197-3201, 2018.

36. Hao H, Liu L, Zhang D, Wang C, Xia G, Zhong F and Hu X: Diagnostic and prognostic value of miR-106a in colorectal cancer. Oncotarget 8: 5038-5047, 2017.

37. Li Y, Zhang Y, Yao Z, Li S, Yin Z and Xu M: Forkhead box Q1: A key player in the pathogenesis of tumors (Review). Int J Oncol 49: 51-58, 2016.

38. Qiao Y, Jiang X, Lee ST, Karuturi RK, Hooi SC and Yu Q: FOXQ1 regulates epithelial-mesenchymal transition in human cancers. Cancer Res 71: 3076-3086, 2011.

This work is licensed under a Creative Commons Attribution-NonCommercial-NoDerivatives 4.0 International (CC BY-NC-ND 4.0) License. 